\title{
Surgical Treatment of Severe Bowel Obstruction as a Rare Complication Following Allogenic Hematopoietic Stem Cell Transplantation
}

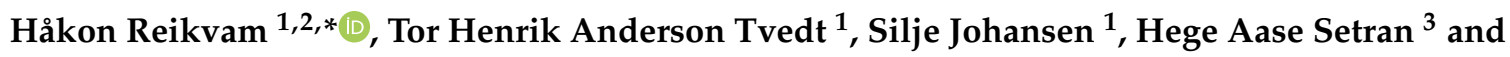 \\ Roald Flesland Havre 1,4(D) \\ 1 Department of Medicine, Haukeland University Hospital, N-5021 Bergen, Norway; \\ tor.henrik.anderson.tvedt@helse-bergen.no (T.H.A.T.); Silje.Johansen@helse-bergen.no (S.J.); \\ roald.flesland.havre@helse-bergen.no (R.F.H.) \\ 2 Department of Clinical Science, University of Bergen, N-5021 Bergen, Norway \\ 3 Department of Pathology, Haukeland University Hospital, N-5021 Bergen, Norway; \\ hege.aase.setran@helse-bergen.no \\ 4 Department of Clinical Medicine, University of Bergen, N-5021 Bergen, Norway \\ * Correspondence: hakon.reikvam@uib.no; Tel./Fax: +47-55-97-50-00
}

Received: 20 October 2020; Accepted: 12 November 2020; Published: 17 November 2020

\begin{abstract}
Gastrointestinal graft-versus-host disease (GVHD) and cytomegalovirus (CMV) disease are common complications occurring after allogeneic hematopoietic stem cell transplantation (allo-HSCT), and contribute to a high degree of morbidity and mortality associated with allo-HSCT. Herein, we present a patient with severe intestinal GVHD complicated by recurring CMV enteritis, which overall resulted in severe terminal ileum stenosis. The patient underwent laparoscopic ileocecal resection that significantly reduced symptoms and possibly prevented the development of fulminant ileus. Surgical treatment is rarely used in the treatment of gastrointestinal GVHD; however, the current patient history illustrates that patients with inadequate symptom control and severe inflammatory bowel stenosis can be successfully managed with surgery. We also review published case reports on surgical treatment for severe gastrointestinal GVHD.
\end{abstract}

Keywords: allogenic hematopoietic stem cell transplantation; GVHD;CMV-disease; bowel obstruction

\section{Introduction}

Graft-versus-host disease (GVHD) is a severe complication seen after allogeneic hematopoietic stem cell transplantation (allo-HSCT) [1,2]. GVHD is an immune response where immunocompetent lymphocytes derived from the donor reject different organ systems in the recipient [1,2]. GVHD is classified based on clinical symptoms and the time of onset. Classical acute GVHD (aGVHD) typically occurs during the first 100 days posttransplant and manifest as hyperbilirubinemia, an acute inflammation of skin and/or mucous membranes, while chronic GVHD (cGVHD) typically occurs after day 100 and has no clinical features of aGVHD. Furthermore, an overlap syndrome has features of both acute and chronic GVHD [1,2]. Traditionally, GVHD has been treated with immunosuppressive agents such as cyclosporine, corticosteroids, tacrolimus, or mycophenolate mofetil [3]. However, since treatment with immunosuppressive agents results in a significant increase in severe and sometimes fatal infection, treatment modalities with less immunosuppressive potential have been developed-i.e., extracorporeal photopheresis (ECP) and mesenchymal stem cells [4,5]. Nevertheless, steroid refractory GVHD has often a lower response rate to second line treatment, and fulminant GVHD has a dismal prognosis. Immunosuppressive therapy in GVHD increases the risk of infectious complications [6], especially cytomegalovirus (CMV) reactivation or primary CMV disease [7]. With regular monitoring for CMV 
and pre-emptive treatment, severe CMV disease are seldom observed [7]. However, recurrent CMV disease in the GI-tract, such as CMV enteritis, can be severe and may require long-term antiviral treatment that is often associated with side effects requiring prolonged hospitalization [7]. In some circumstances, other interventions including surgery may be required. Here, we present a patient with severe and obstructive bowel pathology after allo-HSCT, where surgical intervention was considered the only option for treatment of the patient's symptoms and a review of the literature regarding surgical treatment of GI-tract complications after allo-HSCT.

\section{Case Report}

A 60-year-old man with known history of coronary heart disease developed increasing anemia, and eventually progressive thrombocytopenia and neutropenia. Bone marrow aspiration and biopsy showed signs of myelodysplastic syndrome (MDS) with coexisting fibrosis of the bone marrow. The bone marrow blast percentage was estimated to be $10 \%$. A cytogenetic study showed male karyotype with deletion of the long arm on chromosome 20: 46, XY, del20q. Molecular analysis demonstrated RUNX1 and ASXL1 mutations, both associated with high risk for relapse after allo-HSCT [8]. Treatment with the hypomethylating agent 5 -azacitidine was initiated. After three cycles of 5-azacitidine, the patient achieved reduction in blast count to $<5 \%$, although with persistent pancytopenia. He was accepted for allo-HSCT, and this was performed with fludarabine and treosulfan as a conditioning regimen, before infusion of peripherally derived stem cells from a male unrelated donor with a 10/10 human leukocyte antigen (HLA) match. Both patient and donor were CMV serologically positive. GVHD prophylaxis consisted of antithymocyte globulin (ATG) on day -2 and -1 ; cyclosporin A from day -1 , and methotrexate on days 1, 4, and 6 posttransplant (Figure 1). Engraftment was observed at day 14 posttransplant, and he was dismissed at day 15 .

At day 30, the patient developed a maculopapular rash, clinically and histopathological consistent with aGVHD, Glucksberg grade I. A complete resolution was observed after treatment with local steroids. At day 52, the patient developed abdominal pain and pronounced diarrhea with up to 15 watery stools a day. Due to suspicion of severe aGVHD, a colonoscopy was performed and high-dose treatment with methylprednisolone $2 \mathrm{mg} / \mathrm{kg}$ initiated (Figure 1). There was no sign of liver or skin GVHD, and colon biopsies verified aGVHD; hence, the patient was classified to aGVHD Glucksberg grade IV. The patient's condition improved rapidly, with significant reduction in stool volume, and the treatment could be switched to oral corticosteroid (Figure 1). At day 64, however, an increase in CMV-DNA copies in blood (measured by quantitative polymerase chain reaction, qPCR) was observed, and preemptive treatment with the antiviral agent ganciclovir was initiated. CMV viremia peaked at 11,800 copies/mL, before rapidly decreasing. After two weeks of intravenous twice daily ganciclovir, two weeks of once daily ganciclovir and one week per oral treatment with valganciclovir CMV treatment were stopped (Figure 1). 


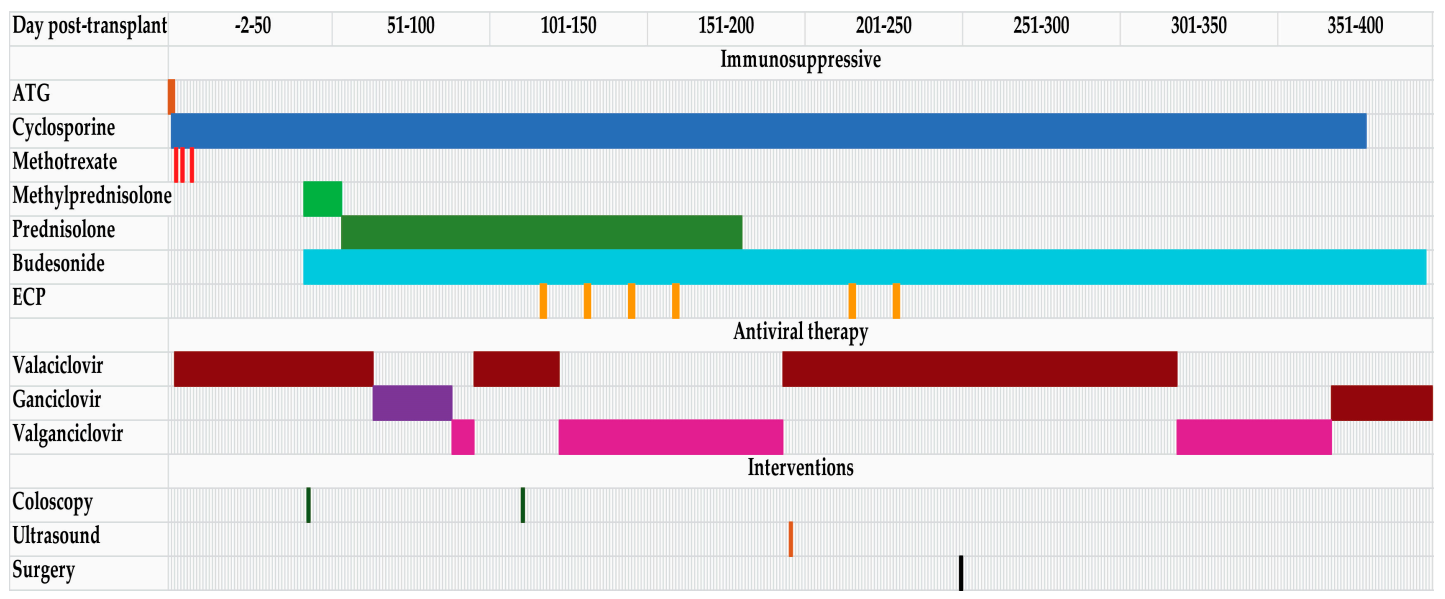

Figure 1. Immunosuppressive, antiviral treatment, and interventions during the posttransplantation course. The figure displays the different immunosuppressive and antiviral therapy during the posttransplantation course. Posttransplant day is given in the top of the figure, and the different colors indicate on which days the patients received the different interventions. Abbreviation: ATG, antithymocyte globulin; ECP, extracorporeal photopheresis.

Diarrhea decreased significantly, with normal shape and consistency of the stool. However, the patient gradually developed intense abdominal pain which required opioid analgesics. The pain was pronounced in the evening, not related to food intake, or passing of flatus or feces. A new colonoscopy was performed, demonstrating active inflammation and ulcerations with granulation tissue within the colon, most pronounced distally in the ileocecal transition, with signs of increasing stenosis in the terminal ileum. Biopsy verified active inflammation, but nuclear CMV was also detected on immunohistochemistry. Immunosuppressive treatment was continued and antiviral treatment with valganciclovir was again initiated. To be able to reduce immunosuppressive therapy-i.e., foremost corticosteroids-ECP was initiated. The patient again experienced a transient improvement in symptoms with decreased pain, almost normal frequency, and form of defecation. Prednisolone could be tapered gradually, and valganciclovir treatment was stopped. Eventually, he developed increasing intermittent abdominal pain that required opioid analgesics. Transabdominal ultrasound examination performed at day +195 (Figure 1) demonstrated an approximately $12 \mathrm{~cm}$ long segment with variegated and markedly increased wall thickness $(5-7 \mathrm{~mm})$ in the terminal ileum (Figure 2). The segment encompassed the entire circumference with total lack of peristaltic movement. These findings of a significant thickened bowel wall in the terminal ileum with signs of narrowing of the lumen were also confirmed by computer tomography (CT) scanning (Figure 3). 
A

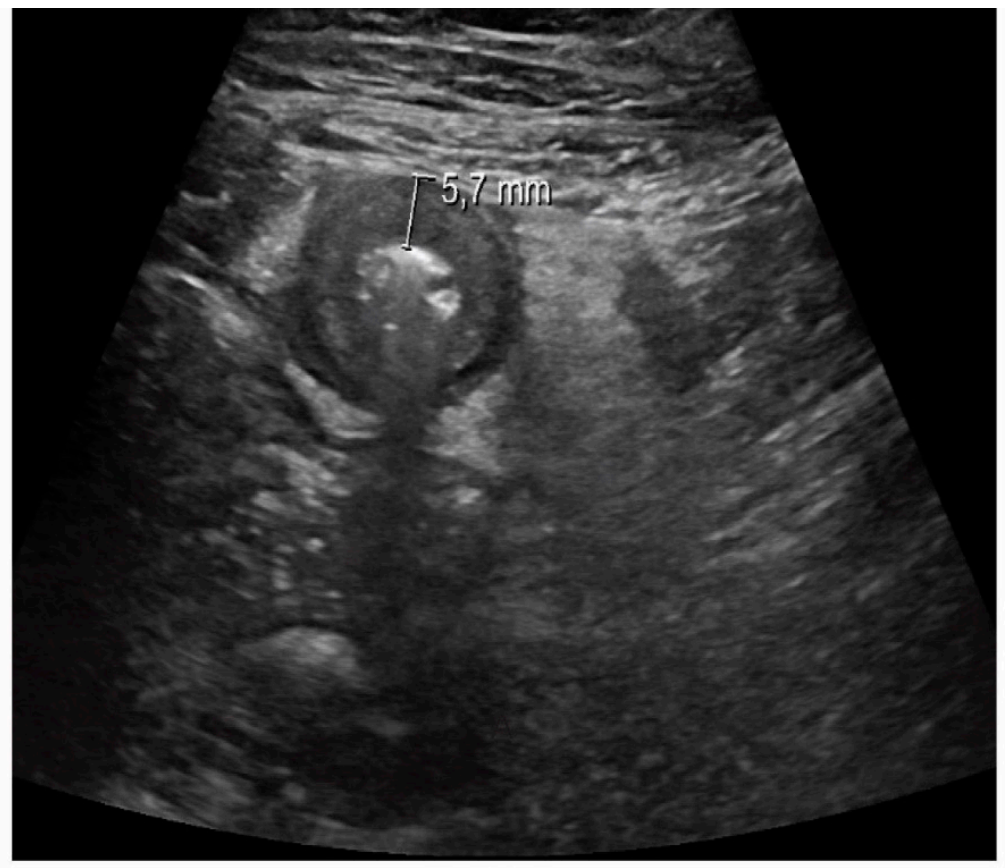

B

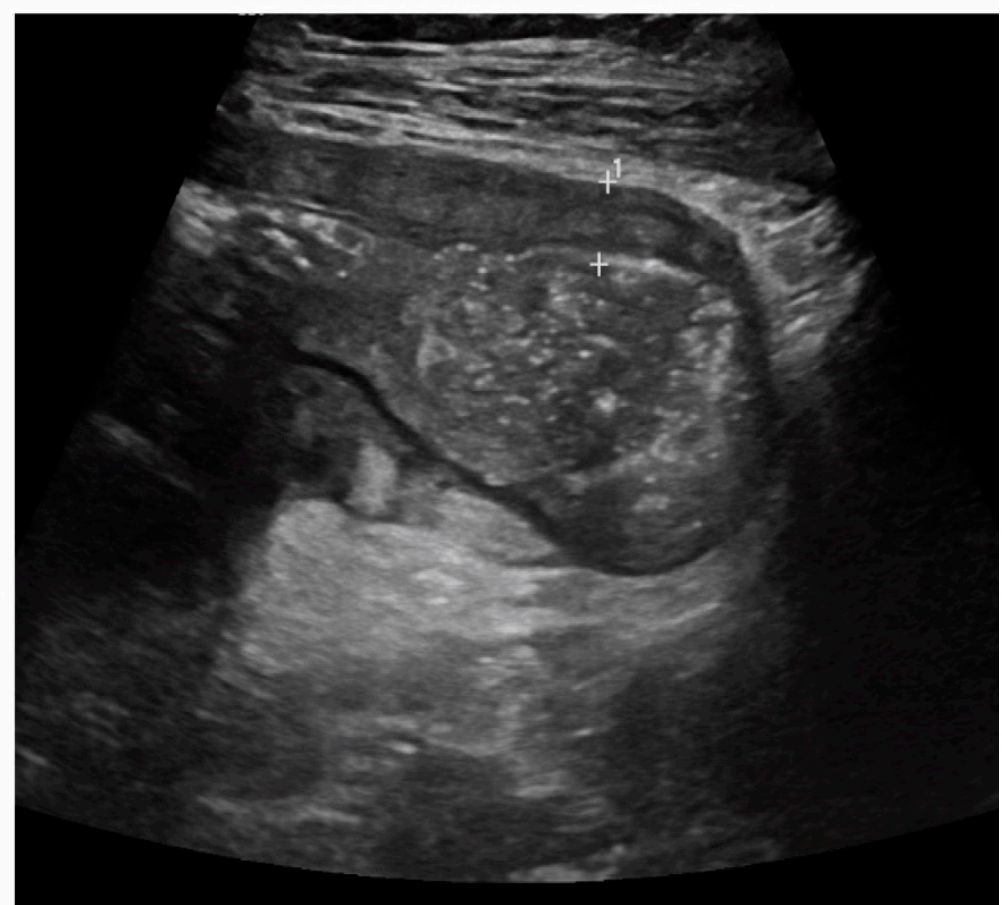

Figure 2. External abdominal ultrasound (US) of the terminal ileum. (A) External cross section of terminal ileum with thickened wall $(5.7 \mathrm{~mm})$ and "creeping fat" surrounding the small intestine circumference. (B) External US of longitudinal/oblique section of the terminal ileum, the bowel wall is markedly thickened, although the mucosa layer is intact. Lumen diameter is wider towards the right indicating a prestenotic dilatation. "Creeping fat" seen below the bowel segment as a hyperechoic area. 


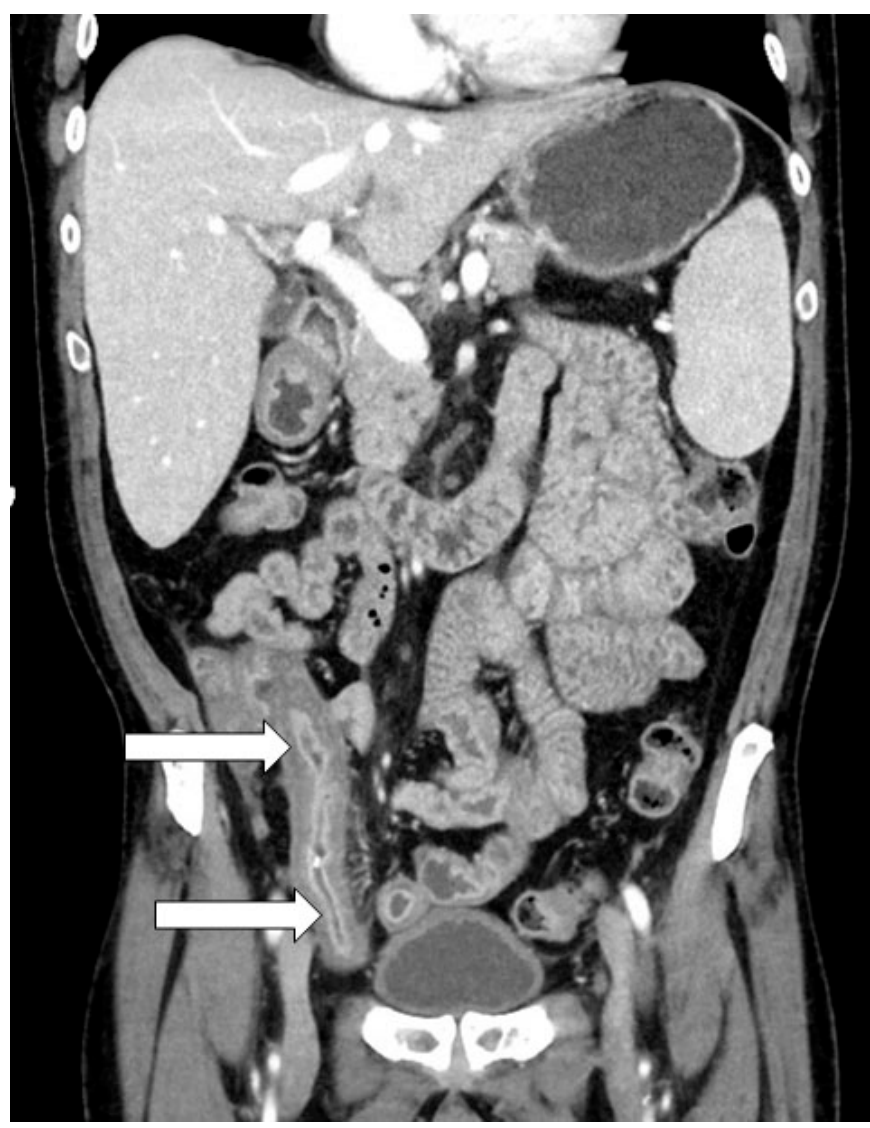

Figure 3. Abdominal computer tomography (CT) scanning. CT image in the coronary plane demonstrating segment of terminal ileum with thickened wall (arrows).

Based on these findings and given the lack of effect of both conventional immunosuppressive treatment and antiviral treatment, and the potential risk of both bacterial and fungal infections with utterly escalation of immunosuppressive treatment, the patient was accepted for surgical intervention. He underwent surgery with laparoscopic ileocecal resection with intracorporeal anastomosis at day +250 posttransplant. The pre- and postoperative courses were uneventful. The surgical intervention had a striking effect on the patient's abdominal pain, and he could shortly after surgery start taper of opioid analgesics. The patient, however, had persistent diarrhea with loose and frequent stools. On pathological assessment, the resected bowel segment demonstrated active inflammation and signs of active CMV disease (Figure 4), despite no increase in CMV-DNA copies in serum by PCR analysis. Following this evaluation, valganciclovir was reintroduced and immunosuppressive treatment taper was initiated. Both cyclosporine and budesonide could be gradually reduced and terminated before day +400 (Figure 1). The treatment had a striking effect on the patient's clinical conditions with normalization of bowel function, and total regression of abdominal pain. He is currently being followed up at an outpatient clinic and has no signs of recurrence, MDS, CMV, or active GVHD 15 months after the transplant procedure. 
A

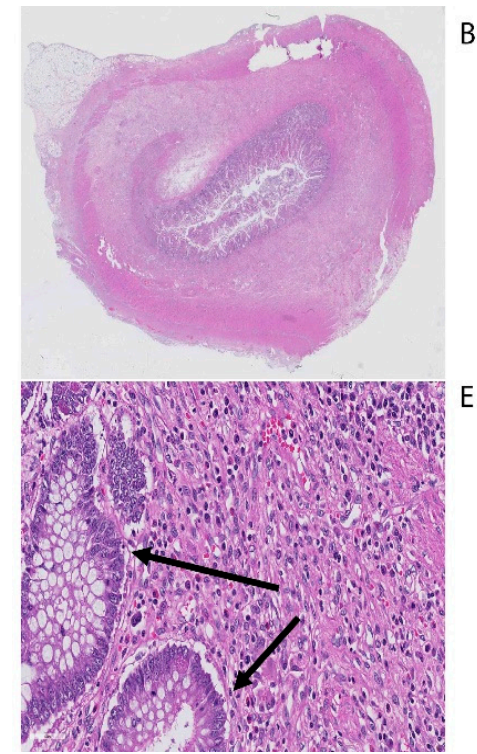

B

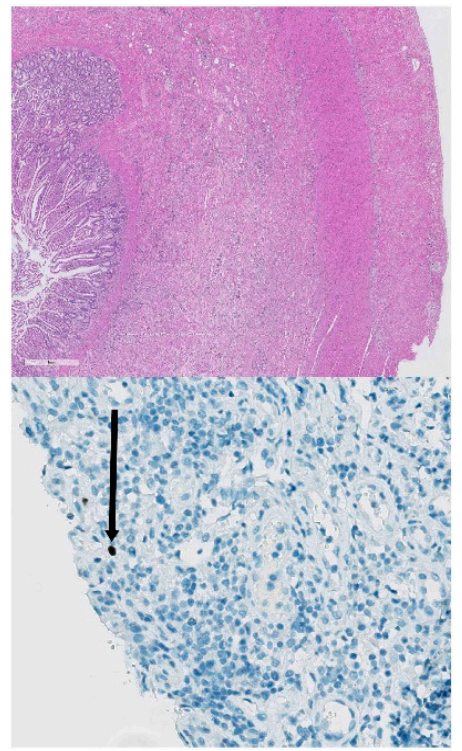

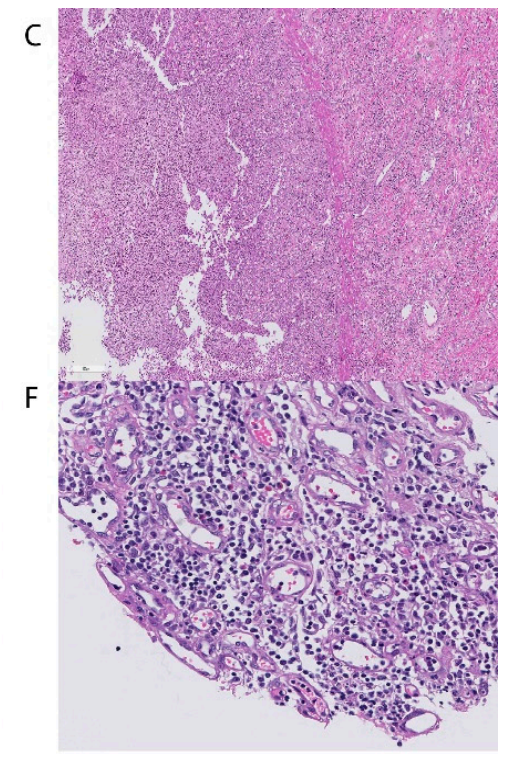

Figure 4. Histopathological examination of the terminal ileum. The figure demonstrates the histopathological findings in biopsies taken at the surgical procedure. (A) Cross section of the terminal ileum showing thickened wall and significantly narrowed lumen, fibrosis, edema, and inflammation. In addition, adipose tissue necrosis and chronic active inflammation and focal ulceration of the mucosa. (B) Cross section from terminal ileum with thickened wall, at higher magnification with severe inflammation. (C) Section from ileum mucosa and submucosa demonstrating pronounced active inflammation of the mucosa with complete loss of crypts and tufts. (D) Higher magnification with apoptotic bodies in crypt epithelium highlighted by arrows. (E) CMV infiltrated cell demonstrated with immunohistochemical staining for CMV (arrow). (F) Section from mucosa with complete loss of tufts and crypts, and with many reactive blood vessels and dense inflammatory infiltrates.

\section{Discussion}

Allo-HSCT is a potent and effective treatment for myeloid malignancies and represents the only real curative treatment for several malignant hematological diseases, such as MDS and acute myeloid leukemia (AML). Unfortunately, an allo-HSCT is associated with significant risk of serious complications, which result in a high degree of morbidity and mortality. Among these complications is steroid resistant or steroid dependent gastrointestinal GVHD. Almost two thirds of patients undergoing allo-HSCT will develop some degree of gastrointestinal aGVHD [9]. Damage to the intestinal mucosa can stimulate an inflammatory response that can induce or enhance the cytokine cascade involved in intestinal aGVHD. Symptoms can develop as isolated intestinal aGVHD, or with concomitant symptoms from other affected organ system such as skin and liver. Risks for the development of GVHD include HLA antigen mismatch, myeloablative conditioning, the use of stem cells derived from blood, and use of an unrelated donor [10]. aGVHD in the intestine usually manifests as abdominal pain and diarrhea and rarely leads to intestinal stricture.

Intestinal GVHD is, according to Glucksberg, divided into stages I through IV, where stages III and IV are associated with a high degree of morbidity and mortality. High doses of corticosteroids are considered first-line treatment, and approximately $70 \%$ of patients will respond to this approach (9). Lack of reduction in stool volumes or progression of symptoms after treatment with steroids after 14 days is defined as steroid refractory GVHD, and the condition is then considered very serious with a poor prognosis [11]. A major side effect of corticosteroid treatment is the risk of viral reactivation, and especially CMV. Molecular-based monitoring for CMV reactivation and preemptive treatment is regarded as standard of care. Concurrent CMV disease and GVHD requiring immunosuppressive therapy is a challenging situation associated with significant mortality, morbidity, and reduced quality of life [7]. Other treatment modalities for intestinal GVHD include pharmacological agents such as 
tacrolimus, mycophenolate mofetil, ruxolitinib, or treatment with ECP. Monoclonal antibodies such as infliximab and vedolizumab have demonstrated promising results in some reports [12-14], although general caution is usually recommended with concomitant CMV or Epstein-Barr virus (EBV) activation. These alternatives were, therefore, considered less appropriate to use in this setting.

As described in the current case report, although the patient initially had severe intestinal aGVHD that seemingly responded to steroid treatment, it was complicated by CMV reactivation, and CMV colitis was also detected. In the present case we believe both GVHD and CMV disease contributed to the severe GI-complications, eventually leading to bowel obstruction. Diagnostic and therapeutic approaches to these well-known complications after allo-HSCT are challenging, and often treatment of both conditions is considered necessary [15]. In our case, we initiated adequate antiviral therapy, and concomitant immunosuppressive therapy was tried tapered when CMV disease was considered. Treatment with ECP was also initiated, which is believed to have more of an immunomodulatory effect than an immunosuppressive effect, and therefore, is associated with less risk of infectious complications [4]. Nevertheless, the patient had persistent abdominal symptoms, and the clinical picture gave suspicion of clinical sub-ileus with significant stenosis. Both colonoscopy and ultrasound of the abdomen verified significant inflammation of a strangulating nature in the terminal ileum similar to findings in Crohn's disease [16]. Conventional GVHD treatment and antiviral treatment were considered inadequate for further therapy, and the obliterating character of the inflammatory process was of such a nature that surgery was considered as the best therapeutic option. Surgical intervention for intestinal GVHD or other post-allo-HSCT complications is rarely necessary, and only a few cases and minor patient material are reported regarding this treatment approach [17-22].

In the published reports, beneficial effects were observed both in child and adult patients with inadequate response to conventional pharmacological treatment. It is noteworthy that bowel obstruction then seems to be the most important indication for surgical treatment, with other indications for surgical intervention also being fistulation, perforation, or bleeding $[17,21]$. Interestingly, the terminal ileum was the predilection site for inflammation and strangulation, which was also the case for our patient. The clinical presentation could be reminiscent of Crohn's disease, where major inflammation, especially in the thermal ileum, may require surgical intervention [16]. The patient also showed evidence of persistent CMV disease in the bowel which was also confirmed in the surgical specimen, and even if CMV is commonly seen as a mucosal infiltration, particularly in the colon, it may have caused the Crohn-like transmural inflammation in this case. The patient had persistent abdominal pain and sub-ileus, while clinical signs of active intestinal inflammation-i.e., diarrhea-decreased. A gastrointestinal contrast study indicated an obstruction in the bowel, and surgery was performed. Successful symptom relief occurred shortly after resection of the obstructed bowel, indicating a clear association between the patient's symptoms and the terminal ileum pathology.

\section{Conclusions}

Severe bowel obstruction is a rare complication seen in patients with GI-complications after allo-HSCT. Conventional pharmacological treatment could be inadequate for these patients. In our case, surgical intervention was a successful treatment option. The present report demonstrates that surgery can be a treatment option in such severe cases, where conventional immunosuppressive treatment regimens have turned out to be negative. However, further clinical studies are warranted to clarify the timing, safety, and effects of surgical interventions for patients with bowel obstruction after allo-HSCT.

Author Contributions: Conceptualization, H.R.; methodology, H.R. and R.F.H.; investigation, H.R., T.H.A.T. and S.J.; resources, H.R., T.H.A.T., S.J., H.A.S. and R.F.H.; writing-original draft preparation, H.R.; writing-review and editing, H.R., T.H.A.T., S.J, H.A.S. and R.F.H.; visualization, H.R., H.A.S. and R.F.H.; supervision, H.R.; project administration, H.R. and R.F.H.; funding acquisition, H.R. All authors have read and agreed to the published version of the manuscript.

Funding: The research related to allo-HSCT was funded by Helse Vest, The Norwegian Cancer Society, Rakel and Otto Kristian Bruuns found, Øyvinn Mølbach-Pedersens found, and Norwegian Society of Internal Medicine. 
Acknowledgments: We are grateful to the patient for their consent to publish this case report.

Conflicts of Interest: The authors declare no conflict of interest related to the manuscript. The funders had no role in the design of the study, interpretation of data, in the writing of the manuscript, or in the decision to publish the case report.

\section{References}

1. Zeiser, R.; Blazar, B.R. Acute graft-versus-host disease-Biologic process, prevention, and therapy. N. Engl. J. Med. 2017, 377, 2167-2179. [CrossRef]

2. Zeiser, R.; Blazar, B.R. Pathophysiology of Chronic graft-versus-host disease and therapeutic targets. N. Engl. J. Med. 2017, 377, 2565-2579. [CrossRef] [PubMed]

3. Penack, O.; Marchetti, M.; Ruutu, T.; Aljurf, M.; Bacigalupo, A.; Bonifazi, F.; Ciceri, F.; Cornelissen, J.; Malladi, R.; Duarte, R.F.; et al. Prophylaxis and management of graft versus host disease after stem-cell transplantation for haematological malignancies: Updated consensus recommendations of the European Society for Blood and Marrow Transplantation. Lancet Haematol. 2020, 7, e157-e167. [CrossRef]

4. Bruserud, Ø.; Tvedt, T.H.A.; Paulsen, P.Q.; Ahmed, A.B.; Gedde-Dahl, T.; Tjønnfjord, G.E.; Slåstad, H.; Heldal, D.; Reikvam, H. Extracorporeal photopheresis (photochemotherapy) in the treatment of acute and chronic graft versus host disease: Immunological mechanisms and the results from clinical studies. Cancer Immunol. Immunother. 2014, 63, 757-777. [CrossRef] [PubMed]

5. Morata-Tarifa, C.; Macías-Sánchez, M.D.M.; Gutiérrez-Pizarraya, A.; Sanchez-Pernaute, R. Mesenchymal stromal cells for the prophylaxis and treatment of graft-versus-host disease-A meta-analysis. Stem Cell Res. Ther. 2020, 11, 64. [CrossRef] [PubMed]

6. Hansen, B.-A.; Wendelbo, Ø.; Bruserud, Ø.; Hemsing, A.L.; Mosevoll, K.A.; Reikvam, H. Febrile neutropenia in acute leukemia. Epidemiology, etiology and treatment. Mediterr. J. Hematol. Infect. Dis. 2019, 12, e2020009. [CrossRef] [PubMed]

7. Ljungman, P.; De La Camara, R.; Robin, C.; Crocchiolo, R.; Einsele, H.; Hill, J.A.; Hubacek, P.; Navarro, D.; Cordonnier, C.; Ward, K.N. Guidelines for the management of cytomegalovirus infection in patients with haematological malignancies and after stem cell transplantation from the 2017 European Conference on Infections in Leukaemia (ECIL 7). Lancet Infect. Dis. 2019, 19, e260-e272. [CrossRef]

8. Della Porta, M.G.; Gallì, A.; Bacigalupo, A.; Zibellini, S.; Bernardi, M.; Rizzo, E.; Allione, B.; Van Lint, M.T.; Pioltelli, P.; Marenco, P.; et al. Clinical effects of driver somatic mutations on the outcomes of patients with myelodysplastic syndromes treated with allogeneic hematopoietic stem-cell transplantation. J. Clin. Oncol. 2016, 34, 3627-3637. [CrossRef] [PubMed]

9. McDonald, G.B. How I treat acute graft-versus-host disease of the gastrointestinal tract and the liver. Blood 2016, 127, 1544-1550. [CrossRef] [PubMed]

10. Grønningsæter, I.S.; Tsykunova, G.; Lilleeng, K.; Ahmed, A.B.; Bruserud, Ø.; Reikvam, H. Bronchiolitis obliterans syndrome in adults after allogeneic stem cell transplantation-pathophysiology, diagnostics and treatment. Expert Rev. Clin. Immunol. 2017, 13, 553-569. [CrossRef]

11. Martin, P.J. How I treat steroid-refractory acute graft-versus-host disease. Blood 2020, 135, 1630-1638. [CrossRef] [PubMed]

12. Rao, K.; Rao, A.; Karlsson, H.; Jagani, M.; Veys, P.; Amrolia, P.J. Improved survival and preserved antiviral responses after combination therapy with daclizumab and infliximab in steroid-refractory graft-versus-host disease. J. Pediatr. Hematol. 2009, 31, 456-461. [CrossRef] [PubMed]

13. Floisand, Y.; Lundin, K.E.A.; Lazarevic, V.; Kristiansen, J.D.; Osnes, L.T.N.; Tjonnfjord, G.E.; Reims, H.M.; Gedde-Dahl, T. Targeting Integrin alpha4beta7 in steroid-refractory intestinal graft-versus-host disease. Biol. Blood Marrow Transplant. 2017, 23, 172-175. [CrossRef]

14. Ross, W. Treatment of gastrointestinal acute graft-versus-host disease. Curr. Treat. Options Gastroenterol. 2005, 8, 249-258. [CrossRef]

15. Einsele, H.; Ljungman, P.T.; Boeckh, M.J. How I treat CMV reactivation after allogeneic hematopoietic stem cell transplantation. Blood 2020, 135, 1619-1629. [CrossRef] [PubMed]

16. Torres, J.; Mehandru, S.; Colombel, J.F.; Peyrin-Biroulet, L. Crohn's disease. Lancet 2017, 389, 1741-1755. [CrossRef] 
17. Gutierrez, C.A.; Raval, M.V.; Vester, H.R.; Chaudhury, S.; Von Allmen, D.; Rothstein, D. Surgical treatment of intestinal complications of graft versus host disease in the pediatric population: Case series and review of literature. J. Pediatr. Surg. 2017, 52, 1718-1722. [CrossRef]

18. Irani, J.L.; Cutler, C.S.; Whang, E.E.; Clancy, T.E.; Russell, S.; Swanson, R.S.; Ashley, S.W.; Zinner, M.J.; Raut, C.P. Severe acute gastrointestinal graft-vs-host disease. Arch. Surg. 2008, 143, 1041-1045. [CrossRef]

19. Gavel, G.; Marven, S.; Evans, M.J.; Walker, J.P.; Vora, A. Obliterative enteritis complicating graft versus host disease. Bone Marrow Transplant. 2003, 32, 1097-1098. [CrossRef]

20. Cornell, R.F.; Palmer, J.; Komorowski, R.; Drobyski, W.R. Surgical resection as definitive treatment for refractory GVHD of the colon. Bone Marrow Transplant. 2012, 47, 1366-1367. [CrossRef]

21. Chirletti, P.; Caronna, R.; Arcese, W.; Iori, A.P.; Calcaterra, D.; Cartoni, C.; Sammartino, P.; Stipa, V. Gastrointestinal emergencies in patients with acute intestinal graft-versus-host Disease. Leuk. Lymphoma 1998, 29, 129-137. [CrossRef] [PubMed]

22. Turki, A.T.; Bayraktar, E.; Basu, O.; Benkö, T.; Yi, J.-H.; Kehrmann, J.; Tzalavras, A.; Liebregts, T.; Beelen, D.W.; Steckel, N.K. Ileostomy for steroid-resistant acute graft-versus-host disease of the gastrointestinal tract. Ann. Hematol. 2019, 98, 2407-2419. [CrossRef] [PubMed]

Publisher's Note: MDPI stays neutral with regard to jurisdictional claims in published maps and institutional affiliations.

(C) 2020 by the authors. Licensee MDPI, Basel, Switzerland. This article is an open access article distributed under the terms and conditions of the Creative Commons Attribution (CC BY) license (http://creativecommons.org/licenses/by/4.0/). 